\title{
Performance Comparison of Category 5e vs. Category 6 Cabling Systems for both IPv4 and IPv6 in Gigabit Ethernet
}

\author{
Burjiz. K. Soorty, Samad. S. Kolahi, Zhang Qu and Navneet Chand \\ UNITEC New Zealand \\ skolahi@unitec.ac.nz \\ sorrtb01@studentmail.unitec.ac.nz
}

\begin{abstract}
The performance of category $5 \mathrm{e}$ and 6 cabling is compared for both IPv4 and IPv6 using Gigabit Ethernet LAN. The maximum bandwidth achieved was $700 \mathrm{Mbps}$ and it was for IPv4 and category 5e cabling.
\end{abstract}

\section{INTRODUCTION}

New technology and a rise in higher bandwidth requirements have inevitably led to a requirement for better cabling systems. CAT 6 is new cabling system at approximate bandwidth of 200 $\mathrm{MHz}$ at $20{ }^{\circ} \mathrm{C}$ for a 100 meter that nearly double of CAT 5e which carries a bandwidth of $100 \mathrm{MHz}$ [1]. Whilst theoretically CAT 6 may well provide better performance for more than a $40 \%$ price premium to the CAT $5 \mathrm{e}$, in reality most computers and networking equipment only transmit a range of frequency of $100 \mathrm{MHz}$. A wide range of tests carried out by various manufacturers however clearly show that currently available CAT 5e applications such as, file transfer, and LAN video streaming over UTP, run markedly better over CAT 6 systems [1]. Despite this several companies still choose to stay with CAT 5e reportedly due to the problems caused by CAT 6 and likely since the costs in upgrade do not justify the minimal gain over Fast and Gigabit Ethernet Networks. CAT 6 being rated at $10 \mathrm{Gbps}$ up to $55 \mathrm{~m}$ and CAT 6 a rated at $10 \mathrm{Gbps}$ up to $100 \mathrm{~m}$ with CAT 5e rated at $1 \mathrm{Gbps}$ up to $55 \mathrm{~m}$ leaves CAT 6 as a viable upgrade mainly for long term investment of 10Gbps Gigabit Ethernet, which is still under development or for existing networks running Gigabit Ethernet over larger distances.

As networks enhance with the rapid advancement in technology and with applications such as VOIP, growth in IP addresses have effectively increased alongside higher bandwidth requirements. The inadequacy of IP addresses is successfully overcome by IPv6 which supports a total of $2^{128}$ addresses as opposed to $2^{32}$ for IPv4 whereas high bandwidth requirements for QOS related applications have in turn led most large networks to transit into an extensive upgrade from Fast Ethernet to Gigabit Ethernet LAN's. As this LAN transition is currently occurring with most large networks and eventually evident with the arrival of newer Ethernet standards a great emphasis is given on the cabling systems as transmission performance remains one of the core domains of any corporate network.
In 2006, Galen Udell from Belden CDT Networking [1] conducted a study on Category 6 vs. Category 5e cabling systems and implications for Voice over IP networks. Their study measured cabling performance through a series of measurements based on inputs to and outputs from the "channel" also known as the "link segment" by IEEE which represents the physical link between the local and the remote equipment. The parameters considered for their study included Attenuation or Insertion Loss which is the measure of the output signal level (noise level) compared to the input signal level, Near End Crosstalk (NEXT) and Far End Crosstalk (FEXT) which is the measure of internal noise generated between pairs within the same cable or connector and, Return Loss which is the measure of "self generated" noise on a given pair due to component impedance mismatches or due to impedance variations along the cable. Their study also measured the speed of the channel by evaluating frequency range and usable bandwidth. The study concluded that the network performance eventually boils down to the Signal-to-Noise Ratio at the Receiver. All the different noise sources needed to be taken into account, including NEXT, FEXT, Signal reflections, Alien Crosstalk and Impulse noise. The biggest benefit of Category 6 cabling was the muchimproved Signal-to-Noise Ratio (SNR) using the Bandwidth employed by today's applications and also for future applications. The main result was that Category 6 provided about $12 \mathrm{~dB}$ (or 16 times) better Signal-to-Noise Ratio compared to Category 5 e over a wide frequency range.

Prior to the above research, another study was performed by Bell Laboratories in 2003 that conducted experiments utilizing three high speed bandwidth intensive applications. The applications chosen were 270 Mbps Serial Digital Video, 100BASE-TX streaming video and 100BASE-TX data file transfer. Their results showed Category 6 cabling solutions provided measurably better throughput performance than solutions compliant with the Category 5e standards [1].

Both studies were based on how the cabling affected the QOS. They concluded that current QOS applications running at $1 \mathrm{Gbps}$ were pushing the limits of Category 5e cabling and with streaming media applications such as video and multi-media becoming more common, the demands for faster data rates would continue to increase and spawn new applications that 
would eventually benefit from the higher bandwidth offered by Category 6.

Our study is based on the performance of IPv4 and IPv6 stack over the two different cabling systems on Gigabit Ethernet LAN's. The different parameters taken into account were the two pre-eminent transport layer protocols, namely the connectionless UDP generally used for VOIP applications and the connection-oriented TCP used for reliable data transfer. The performance metric taken into account was the Round Trip Time or latency to measure the delay caused by packet loss. RTT was measured since packet loss is a big problem for VOIP applications as it results in degraded voice quality. The cabling system can directly be attributed to packet loss if the number of collisions increases due to bandwidth being close to near-high capacity thereby resulting in overloaded links [1].

Another performance metric taken into account was throughput. When data throughput is high, the Bit Error Rate (BER) is low, thus improving Return Loss (Impedance Variations) on cabling systems [1]. Packet size was another parameter implemented in this evaluation as higher packet sizes would result in fewer acknowledgements for TCP thereby affecting overall throughput and latency.

\section{NETWORK SETUP}

The hardware benchmark comprised of an Intel ${ }^{\circledR}$ Core ${ }^{\mathrm{TM}} 2$ Duo $63001.87 \mathrm{GHz}$ processor with 2.00 GB RAM for the efficient operation of Windows Vista, a Broadcom NetXtreme Gigabit Ethernet NIC, a Western Digital Caviar SE 160 GB hard-drive on the two workstations and a Category 5e and Category 6 crossover cable in TIA/EIA 568-B wiring to maintain global industrial networking standards for use over 1000Base-T networks.

Figure 1: Network Test-Bed

The proposed network setup involved two test-beds as shown

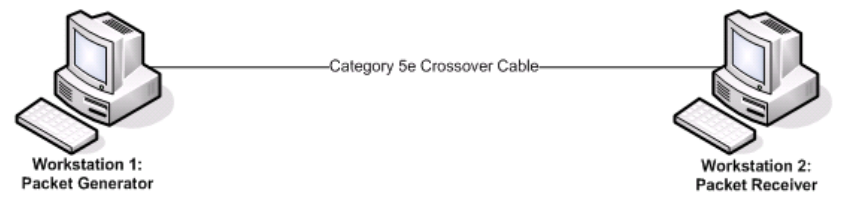

in figure 1.1, the first of which involved setting up a direct connection via standard Category 5e cabling between two workstations and the second test-bed which set a direct connection via standard Category 6 cabling between two workstations. This was done in order to calculate the raw throughput and RTT without the use of a hub, switch or a router that could create latency and degrade the actual speed of the network.

Following are the performance specifications of the respective Category 5e and Category 6 UTP cables used:

\begin{tabular}{|c|c|}
\hline \multicolumn{2}{|c|}{ Category 5e UTP Cable [2] } \\
\hline Spark Test & $2000 \pm 250 \mathrm{~V} \mathrm{ac}$ \\
\hline Dielectric Strength & $2500 \mathrm{~V} \mathrm{dc} / 3 \mathrm{~seconds}$ \\
\hline Insulation Resistance Test & Min. $150 \mathrm{M} \Omega / \mathrm{Km}$ \\
\hline
\end{tabular}

\begin{tabular}{|c|c|c|c|c|}
\hline \multicolumn{2}{|c|}{ Conductor Resistance } & \multicolumn{3}{|c|}{ Max. $9.38 \Omega / 100 \mathrm{~m}$ at $20^{\circ} \mathrm{C}$} \\
\hline \multicolumn{2}{|c|}{ Resistance Unbalance } & \multicolumn{3}{|c|}{ Max. 2\% } \\
\hline \multicolumn{2}{|c|}{ Capacitance Unbalance } & \multicolumn{3}{|c|}{ Max. $160 \mathrm{pF} / 100 \mathrm{~m}$} \\
\hline \multicolumn{2}{|c|}{ Mutual Capacitance } & \multicolumn{3}{|c|}{ Max. $5600 \mathrm{pF} / 100 \mathrm{~m}$} \\
\hline \multirow{2}{*}{ Impedance } & $722 \mathrm{kHz}$ & \multirow{2}{*}{\multicolumn{3}{|c|}{$102 \Omega \pm 15 \%$}} \\
\hline & $1 \sim 125 \mathrm{MHz}$ & & & \\
\hline \multirow{13}{*}{$\begin{array}{c}\text { Attenuation } \\
\text { and Near } \\
\text { End Cross } \\
\text { Talk }\end{array}$} & $\begin{array}{c}\text { Frequency } \\
(\mathrm{MHz})\end{array}$ & $\begin{array}{c}\text { Attenuation } \\
(\mathrm{dB} / 100 \mathrm{M} \\
\left.\text { at } 20^{\circ} \mathrm{C}\right), \\
\operatorname{Max}\end{array}$ & $\begin{array}{c}\text { NEXT } \\
(\mathrm{dB}), \\
\text { Min }\end{array}$ & $\begin{array}{c}\begin{array}{c}\text { Power } \\
\text { Sum } \\
(\mathrm{dB}), \\
\text { Min }\end{array}\end{array}$ \\
\hline & $722 \mathrm{KHz}$ & -- & 67.0 & 64.0 \\
\hline & $1 \mathrm{MHz}$ & -- & 65.0 & 62.0 \\
\hline & $4 \mathrm{MHz}$ & 4.9 & 56.0 & 53.0 \\
\hline & $8 \mathrm{MHz}$ & 7.0 & 51.0 & 48.0 \\
\hline & $10 \mathrm{MHz}$ & 7.8 & 50.0 & 47.0 \\
\hline & $16 \mathrm{MHz}$ & 9.8 & 47.0 & 44.0 \\
\hline & $20 \mathrm{MHz}$ & 11.1 & 45.0 & 42.0 \\
\hline & $25 \mathrm{MHz}$ & 12.5 & 44.0 & 41.0 \\
\hline & $31.25 \mathrm{MHz}$ & 14.0 & 42.0 & 39.0 \\
\hline & $62.5 \mathrm{MHz}$ & 20.4 & 38.0 & 35.0 \\
\hline & $100 \mathrm{MHz}$ & 26.4 & 35.0 & 32.0 \\
\hline & $125 \mathrm{MHz}$ & 30.0 & 34.0 & 31.0 \\
\hline
\end{tabular}

\begin{tabular}{|c|c|c|c|c|}
\hline \multicolumn{5}{|c|}{ Category 6 UTP Cable [3] } \\
\hline \multicolumn{2}{|c|}{ Spark Test } & \multicolumn{3}{|c|}{$2000 \pm 250 \mathrm{~V} \mathrm{ac}$} \\
\hline \multicolumn{2}{|c|}{ Dielectric Strength } & \multicolumn{3}{|c|}{$2500 \mathrm{~V} \mathrm{dc} / 3$ seconds } \\
\hline \multicolumn{2}{|c|}{ Insulation Resistance Test } & \multicolumn{3}{|c|}{ Min. $150 \mathrm{M} \Omega / \mathrm{Km}$} \\
\hline \multicolumn{2}{|c|}{ Conductor Resistance } & \multicolumn{3}{|c|}{ Max. $9.38 \Omega / 100 \mathrm{~m}$ at $20^{\circ} \mathrm{C}$} \\
\hline \multicolumn{2}{|c|}{ Resistance Unbalance } & \multicolumn{3}{|c|}{ Max. $2 \%$} \\
\hline \multicolumn{2}{|c|}{ Capacitance Unbalance } & \multicolumn{3}{|c|}{ Max. $160 \mathrm{pF} / 100 \mathrm{~m}$} \\
\hline \multicolumn{2}{|c|}{ Mutual Capacitance } & \multicolumn{3}{|c|}{ Max. $5600 \mathrm{pF} / 100 \mathrm{~m}$} \\
\hline \multirow{2}{*}{ Impedance } & $64 \mathrm{kHz}$ & \multicolumn{3}{|c|}{$125 \Omega \pm 20 \%$} \\
\hline & $1 \sim 250 \mathrm{MHz}$ & \multicolumn{3}{|c|}{$100 \Omega \pm 15 \%$} \\
\hline \multirow{12}{*}{$\begin{array}{c}\text { Attenuation } \\
\text { and Near } \\
\text { End Cross } \\
\text { Talk }\end{array}$} & $\begin{array}{c}\text { Frequency } \\
(\mathrm{MHz})\end{array}$ & $\begin{array}{c}\text { Attenuation } \\
(\mathrm{dB} / 100 \mathrm{M} \\
\left.\text { at } 20^{\circ} \mathrm{C}\right) \\
\operatorname{Max}\end{array}$ & $\begin{array}{c}\text { NEXT } \\
(\mathrm{dB}), \\
\text { Min }\end{array}$ & $\begin{array}{c}\begin{array}{c}\text { Power } \\
\text { Sum } \\
(\mathrm{dB}), \\
\text { Min }\end{array} \\
\end{array}$ \\
\hline & $1 \mathrm{MHz}$ & -- & 74.3 & 64.0 \\
\hline & $4 \mathrm{MHz}$ & 3.8 & 65.3 & 63.3 \\
\hline & $10 \mathrm{MHz}$ & 6.0 & 59.3 & 57.3 \\
\hline & $16 \mathrm{MHz}$ & 7.6 & 56.2 & 54.2 \\
\hline & $20 \mathrm{MHz}$ & 8.5 & 54.8 & 52.8 \\
\hline & $31.25 \mathrm{MHz}$ & 10.7 & 51.9 & 49.9 \\
\hline & $62.5 \mathrm{MHz}$ & 15.5 & 47.4 & 45.4 \\
\hline & $100 \mathrm{MHz}$ & 19.9 & 44.3 & 42.3 \\
\hline & $150 \mathrm{MHz}$ & 25.3 & 41.4 & 39.4 \\
\hline & $200 \mathrm{MHz}$ & 29.2 & 39.8 & 37.8 \\
\hline & $250 \mathrm{MHz}$ & 33.0 & 38.3 & 36.3 \\
\hline
\end{tabular}

The two workstations were connected by a distance of an approximate one meter which is the average distance between computes in a medium to large corporate network. The length of the Category 5e and Category 6 cables were measured to be 
equal in size at 2 meters in length. Both cables used were ISO 9001 certified and were produced by the same manufacturing company called YFC.

The operating system installed was Microsoft Windows Vista (plus Service Pack One).

\section{Data Generation and Traffic Measurement Tool}

IP Traffic [4] was selected as the traffic generating and measurement tool for its compatibility with Windows Vista, and for its powerful analysis of a wide range of quality of service parameters to acquire accurate results. IP Traffic was the primary tool used for measuring IPv4 and IPv6 performance on Windows XP over Fast Ethernet [5]. Furthermore, IP Traffic has extensively been used for various other researches including performance evaluation of network security [6] and impact of encryption effects on network performance [7].

\section{RESULTS}

The TCP and UDP throughput and RTT were measured for IPv4 and IPv6 for various packet sizes. The range of packet sizes varied from 128 to 1408 bytes over two Peer to Peer networks running Windows Vista operating system, one connected via Category 5e and the other through a Category 6 cable.

This evaluation methodology comprised of performing 40 test runs for every protocol individually (TCP and UDP) and for each specific packet size (128 to 1408) in-order to get rid of any inconsistencies shown in the results. One run included sending 1 million packets of one particular packet size and protocol.

Figure 2 shows the TCP throughput for IPv4 and IPv6 for Windows Vista on CAT 5e and CAT 6. The most distinctive difference observed between the two cabling systems is that CAT 5e results in higher throughput for IPv6 than on IPv4 for packet sizes $128-896$ bytes whereas the relatively new standard of CAT 6 cabling consistently provides higher throughput for IPv4 than on IPv6 for all packet sizes. Throughput on CAT 5e also shows a steady increase for IPv4 and IPv6 as compared to CAT 6 where the growth in throughput varies with the increase in each packet size. Similarly the difference in IPv4 and IPv6 throughput is not as significant in CAT 5e as observed in CAT 6, especially at packet size 896 bytes for the latter where the growth in throughput on IPv4 is not as high as it shows for IPv6.

Figure 2: TCP Throughput Comparison of IPv4 and IPv6 for Windows Vista on Category 5e vs. Category 6

Comparing the overall performance of the two cabling systems, TCP traffic shows IPv4 and IPv6 to perform by far better with CAT 5e than it does with CAT 6 . This is especially

\section{TCP Throughput Comparison for IPv4 and IPv6}

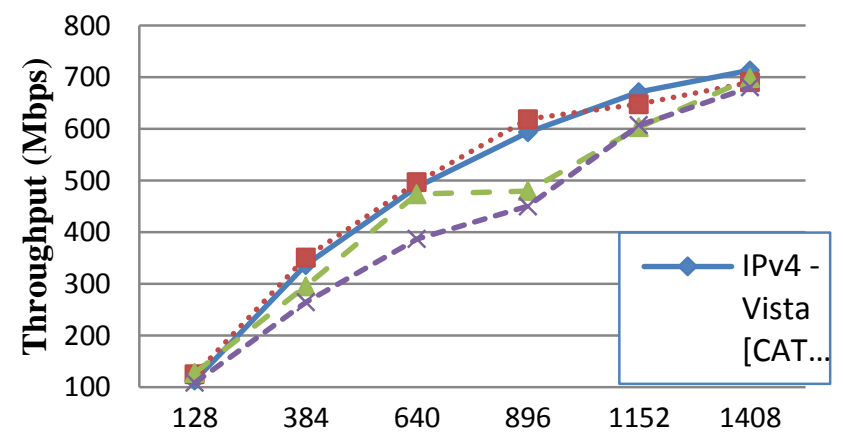

significant with the lower and mid-range packet sizes of 128 896 bytes after which point the gap in difference decreases.
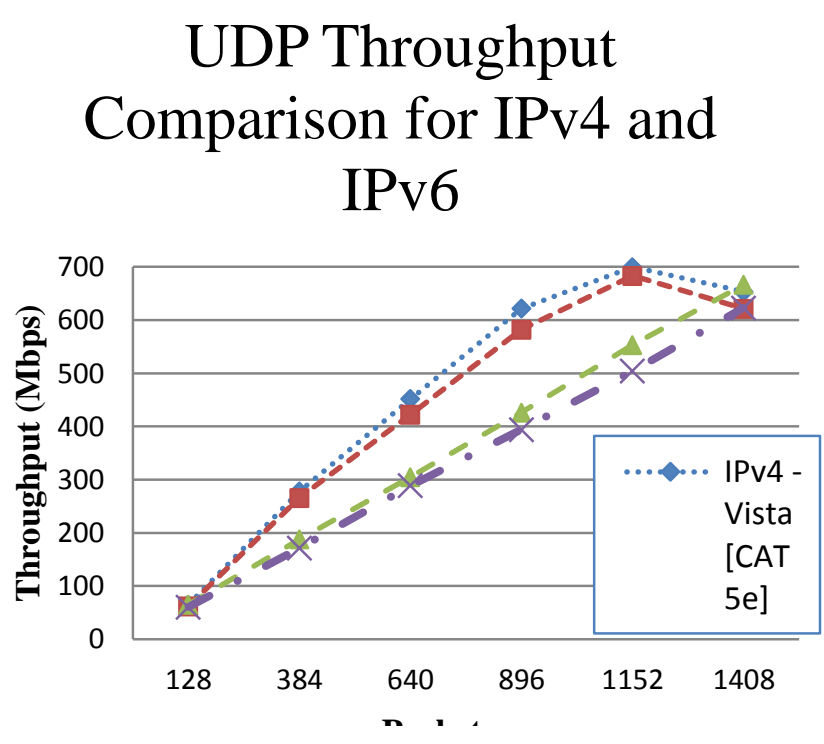

Figure 3: UDP Throughput Comparison of IPv4 and IPv6 for Windows Vista on Category 5e vs. Category 6

Figure 3 shows the UDP throughput for IPv4 and IPv6 for Windows Vista on CAT 5e and CAT 6. As depicted, once again the throughput for IPv4 and IPv6 is considerably higher with CAT 5e than it is with CAT 6. It is interesting to note that CAT 5 e reports a drop in bandwidth for the last packet size resulting with a throughput close to that of CAT 6 . This pattern is consistent as earlier noticed with TCP which show the gap in throughput decrease with the increase in packet size. In both instances, throughput on IPv4 and IPv6 was lower on CAT 6 for the smaller and mid-range packet sizes but close to that of CAT 5e with the higher packet sizes.

Unlike the TCP throughput of IPv4 and IPv6 observed on CAT 5e where IPv6 comparatively reported a higher 
throughput than IPv4, UDP results produce a higher throughput on IPv4 than IPv6 on CAT 5e.

Similarly, unlike the TCP throughput of IPv4 and IPv6 observed on CAT 6 where the gap of difference in throughput relatively decreases between IPv4 and IPv6 for the mid-range and large packet sizes as packet size grows, UDP results show this gap increasing with each packet size.

Windows Vista with CAT 5e showed TCP throughput to be higher on IPv6 than on IPv4 with a maximum difference of 25.43 Mbps showing a $4.28 \%$ increase in IPv6 for the packet size 896 bytes (618.9 Mbps for IPv6 vs. $593.47 \mathrm{Mbps}$ for IPv4). UDP throughput however resulted in higher performance on IPv4 than on IPv6 with a maximum difference of $39.79 \mathrm{Mbps}$ showing a $6.83 \%$ increase in IPv4 for the packet size 896 bytes (621.92 Mbps for IPv4 vs. 582.13 Mbps for IPv6).

Windows Vista with CAT 6 showed TCP throughput to be higher on IPv4 than on IPv6 with a maximum difference of 86.89 Mbps showing a 22.45\% increase in IPv4 for packet size 640 bytes (473.82 Mbps for IPv4 vs. 386.93 Mbps for IPv6). UDP throughput also resulted in higher performance on IPv4 than on IPv6 with a maximum difference of $48.85 \mathrm{Mbps}$ showing a $9.68 \%$ increase in IPv4 for packet size 1152 bytes (553.07 Mbps for IPv4 vs. 504.22 Mbps for IPv6).

Comparing the overall performance of the two cabling systems, IPv4 and IPv6, both resulted in better TCP throughput on CAT 5e than on CAT 6. CAT 5e showed an $11.26 \%$ decrease of $14.38 \mathrm{Mbps}$ in IPv4 throughput from CAT 6 for the lowest packet size of 128 bytes (113.22 Mbps for CAT 5e vs. 127.6 Mbps for CAT 6) and a $2.13 \%$ increase of $14.91 \mathrm{Mbps}$ in IPv4 throughput from CAT 6 for the highest packet size of 1408 bytes (713.18 Mbps for CAT 5e vs. 698.27 Mbps for CAT 6). IPv6 also resulted in better TCP throughput on CAT 5e than on CAT 6 with CAT 5e showing a $14.79 \%$ increase of $16.09 \mathrm{Mbps}$ in IPv6 throughput for the lowest packet size of 128 bytes (124.82 Mbps for CAT 5e vs. 108.73 Mbps for CAT 6) and a $1.44 \%$ increase of $9.81 \mathrm{Mbps}$ in IPv6 throughput from CAT 6 for the highest packet size of 1408 bytes (690.39 Mbps for CAT 5e vs. $680.58 \mathrm{Mbps}$ for CAT 6). The highest point of difference with regards to TCP throughput between the two cabling systems stood at the packet size of 896 bytes for both IPv4 and IPv6. CAT 5e showed a 23.70\% increase of $113.72 \mathrm{Mbps}$ for IPv4 (593.47 Mbps for CAT 5e vs. 479.75 Mbps for CAT 6) and a $37.47 \%$ increase of $168.72 \mathrm{Mbps}$ for IPv6 (618.9 Mbps for CAT 5e vs. $450.18 \mathrm{Mbps}$ for CAT 6) at the aforementioned packet size. In terms of UDP throughput, IPv4 and IPv6 also performed comparatively better with CAT 5e than with CAT 6 despite CAT 5e displaying a $0.5 \%$ decrease of $0.37 \mathrm{Mbps}$ in IPv4

throughput from CAT 6 for the lowest packet size of 128 bytes (65.07 Mbps for CAT 5e vs. 65.44 Mbps for CAT 6) and a 1.95\% decrease of $13.06 \mathrm{Mbps}$ in IPv4 throughput from CAT 6 for the highest packet size of 1408 bytes (653.39 Mbps for CAT 5e vs. 666.45 Mbps for CAT 6). IPv6 also resulted in an overall better UDP throughput on CAT 5e than on CAT 6 with CAT 5e showing a $2.60 \%$ increase of $1.57 \mathrm{Mbps}$ in IPv6 throughput for the lowest packet size of 128 bytes (61.94 Mbps for CAT 5e vs. 60.37 Mbps for CAT 6) and a $0.30 \%$ decrease of $1.88 \mathrm{Mbps}$ in IPv6 throughput from CAT 6 for the highest packet size of 1408 bytes (621.25 Mbps for CAT 5e vs. 623.13 Mbps for CAT 6). The highest point of difference with regards to UDP throughput between the two cabling systems stood also at the packet size of 896 bytes for both IPv4 and IPv6. CAT 5e showed a $45.82 \%$ increase of 195.45 Mbps for IPv4 (621.92 Mbps for CAT 5e vs. 426.47 Mbps for CAT 6) and a $47.65 \%$ increase of 187.89 Mbps for IPv6 (582.13 Mbps for CAT 5e vs. 394.24 Mbps for CAT 6) at the aforementioned packet size.

Figure 4: RTT Comparison of TCP for IPv4 and IPv6 for Windows Vista on Category 5e vs. Category 6

Figure 4 shows the TCP Round Trip Time for IPv4 and IPv6 using CAT 5e and CAT 6. The TCP results show a gain in delay for IPv4 and IPv6 with the increase in each packet size. With CAT 5e, IPv6 has a slightly lower delay than IPv4 on all packet sizes. The highest point of difference between IPv4 and IPv6

RTT Comparison of TCP

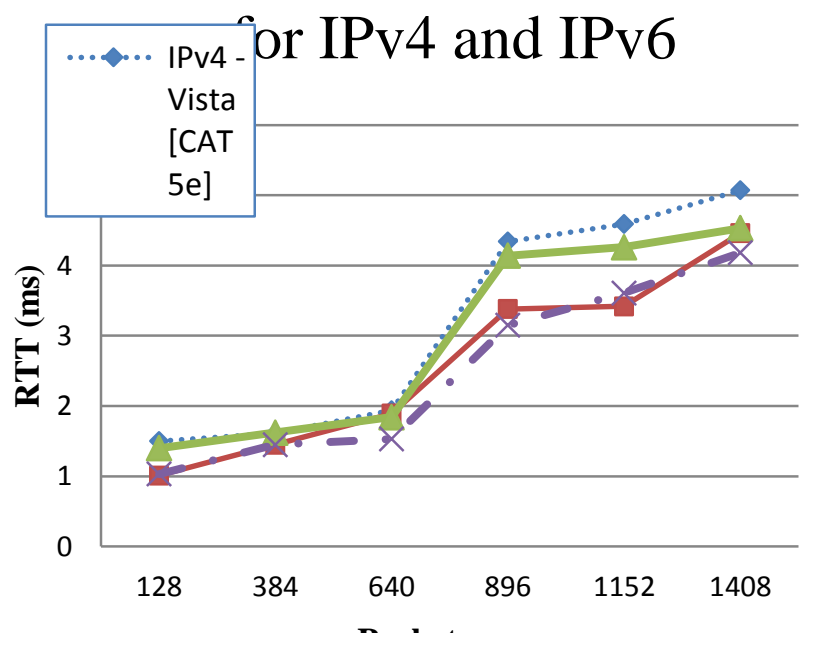

for CAT 5e lies at the packet size of 1152 bytes where IPv6 has a lower delay rate by $1.17 \mathrm{~ms}$ at $34.21 \%$ compared to IPv4 (4.59 ms for IPv4 vs. $3.42 \mathrm{~ms}$ for IPv6). On CAT 6, IPv6 again has a slightly lower delay rate than IPv4. The highest point of difference between IPv4 and IPv6 for CAT 6 stands at the packet size of 896 bytes where IPv6 has a lower delay rate by $0.99 \mathrm{~ms}$ at $31.42 \%$ compared to IPv4 (4.14 ms for IPv4 vs. 3.15 ms for IPv6). 
Comparing the overall performance of the two cabling systems, as depicted, the RTT for TCP is lowest on CAT 6 with IPv6. IPv6 resulted in a slightly lower delay rate overall on CAT 6 than on CAT 5e due to the comparatively low RTT encountered on packet sizes 640, 896 and 1408 bytes as observed. The highest point of difference lied at $6.69 \%$ for a difference of $0.28 \mathrm{~ms}$ for the packet size of 1408 bytes $(4.46 \mathrm{~ms}$ for CAT 5e vs. $4.18 \mathrm{~ms}$ for CAT 6). IPv4 also resulted in a slightly lower delay rate on CAT 6 than on CAT 5e, the maximum difference of which lied at $11.92 \%$ for a difference of $0.54 \mathrm{~ms}$ for the packet size of 1408 bytes (5.07 for CAT 5e vs. $4.53 \mathrm{~ms}$ for CAT 6).

\section{RTT Comparison of UDP}

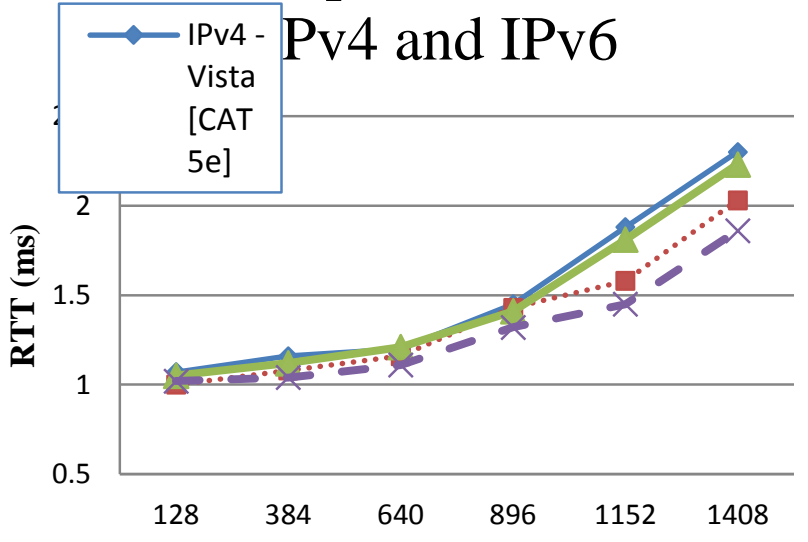

Figure 5: RTT Comparison of UDP for IPv4 and IPv6 for Windows Vista on Category 5e vs. Category 6

Figure 5 shows the UDP Round Trip Time for IPv4 and IPv6 on Windows Vista using CAT 5e and CAT 6. The UDP results once again portray a slow gain in delay for IPv4 and IPv6 as each packet size increases methodically. As the packet size increases towards the mid-range of 640 bytes the gain in delay is increased on both cabling systems. For CAT 5e, the difference in RTT for UDP remains by far insignificant albeit results reveal IPv6 to have a slightly lower delay rate. For CAT 6 , the difference in RTT for UDP remain equally insignificant with IPv6 again displaying a marginally lower delay rate.

Comparing the overall performance of the two cabling systems, the RTT for UDP is lowest on CAT 6 with IPv6, however as observed that difference is largely insignificant. IPv4 resulted in a slightly lower delay rate overall on CAT 6 than on CAT 5e. The highest point of difference lied at $9.85 \%$ for a difference of $0.07 \mathrm{~ms}$ for the packet size of 1408 bytes $(2.3$ for CAT 5e vs. $2.23 \mathrm{~ms}$ for CAT 6). IPv6 also resulted in a marginally lower delay rate on CAT 6 than on CAT 5e, the maximum difference of which lied at $9.13 \%$ for a difference of
$0.17 \mathrm{~ms}$ for the packet size of 1408 bytes $(2.03 \mathrm{~ms}$ for CAT $5 \mathrm{e}$ vs. $1.86 \mathrm{~ms}$ for CAT 6).

Network performance effectively boils down to Signal-toNoise Ratio at the Receiver. All the different noise sources need to be taken into account, including NEXT, FEXT, ILD Noise, Alien Crosstalk and Impulse noise. The biggest benefit of Category 6 cabling is the much-improved Signal-to-Noise Ratio (SNR) using the Bandwidth employed by today's applications and also for future applications. The main result is that Category 6 provides about $12 \mathrm{~dB}$ (or 16 times) better Signal-to-Noise Ratio compared to Category 5 / 5e over a wide frequency range [1] however the raw throughput of IPv4 and IPv6 performance measured in the implemented Category $5 \mathrm{e}$ and Category 6 cabling systems using Windows Vista proved otherwise.

\section{CONCLUSION}

IPv4 and IPv6 both resulted in better TCP throughput with Category 5e than it did with Category 6. IPv4 and IPv6 also resulted in better UDP throughput on Category $5 \mathrm{e}$ than it did with Category 6.

IPv4 and IPv6 resulted in a marginally lower RTT for TCP and UDP with Category 6 than it did with Category 5e. Category 6 cabling might be more suitable for VoIP applications that are delay sensitive.

\section{ACKNOWLEDGMENT}

The authors would like to thank UNITEC Institute of Technology for funding the research team and providing the inventory needed. They are also thankful to Chris Manford for his valuable input and suggestions that helped improve this project.

\section{REFERENCES}

[1] G. Udell, "Category 6 vs. Category 5e Cabling Systems and Implications for Voice over IP Networks," 2006.

[2] YFC-BonEagle ELECTRIC CO., LTD., "CAT.5e UTP," 2007; http://www.yfc-

china.com/yuefeng/en/admin/manage/upload/upfiles/20081222115143.p df.

[3] YFC-BonEagle ELECTRIC CO., LTD., "CAT.6 UTP," 2007; http://www.yfc-

china.com/yuefeng/en/admin/manage/upload/upfiles/200961014359.pdf.

[4] ZTI Telecom, "IP Traffic - test \& measure," http://www.zti-telecom.com.

[5] S. Narayan, S. S. Kolahi, Y. Sonanto, D. T. Nguyen, and P. Mani, "Performance comparison of IPv4 and IPv6 on various windows operating systems," Proc. 11th International Conference on Computer and Information Technology, 2008, pp. 663-668.

[6] N. Baghaei and R. Hunt, "IEEE 802.11 wireless LAN security performance using multiple clients," Proc. Proceedings, The 12th IEEE International Conference on Networks, 2004, pp. 299-303 vol.291.

[7] B. Ezedin, B. Mohammed, A. Amal, S. Hanadi Al, K. Huda, and M. Meera Al, "Impact of Security on the Performance of Wireless-Local Area Networks," Proc. Innovations in Information Technology, 2006, pp. $1-5$. 